\title{
SUPERFÍCIE ESTRIADA POR GELEIRA NEOPALEOZOICA NO PARAGUAI ORIENTAL
}

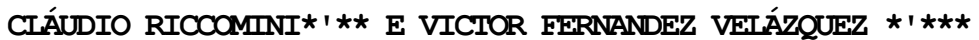

\begin{abstract}
LATE PALEOZOIC GLACIALLY STRIATED SURFACE IN EASTERN PARAGUAY A subhorizontal, completely striated surface, developed upon sedimentary glacial deposite of the Late Paleozoic Aquidabán Formation, has been found in the village of Escobar, Eastern Paraguay. The striae are shallow, parallel grooves a few millimeters apart, rarely deeper than $1 \mathrm{~mm}$, orientated N35E. Locally the surface is covered by diamictite with flat-iron-shaped and striated clasts. The characteristics of the pavement and its association with the diamictite support its origin by glacial abrasion. Transverso fractures associated with the surface indicate ice movement from southwest to northeast. This sense of movement is not consistem with a glacier source centered upon the Asunción Arch, as postulated in previous works. Rather, the data favour a source area located to the south-southeast of the Paraná Basin.

Keywords: striated surface, Aquidabán Formation, Late Paleozoic, glaciation, Eastern Paraguay.

RESUMO Na localidade de Escobar, Paraguai Oriental, foi encontrada uma superficie suborizontal, inteiramente estriada, desenvolvida sobre depósitos areno-rudáceos, de origem glacial, da Formação Aquidabán (Neopaleozóico). As estrias correspondem a sulcos paralelos, de profundidade raramente superior a $1 \mathrm{~mm}$, com espaçamento de alguns milímetros, orientados segundo N35E. Localizadamente a superficie está recoberta por diamictito com seixos facetados e estriados. As características da superfície e sua associação com o diamictito são indicativas de sua origem por abrasão de geleira. Fraturas tranversais associadas à superficie mostram sentido de movimentação do gelo de sudoeste para nordeste. Este sentido não é consistente com uma possível proveniência de centro glacial situado sobre o Arco de Assunção, referida em trabalhos anteriores. Ao contrário, os dados obtidos favorecem a hipótese de proveniência de geleiras a partir de áreas situadas a sul-sudeste da Bacia do Paraná.

Palavrax-chave: superfície estriada, Formação Aquidabán, Neopaleozóico, glaciação, Paraguai Oriental.
\end{abstract}

INTRODUÇ̃̃O A expansão das massas de gelo da glaciação neopaleozóica na Bacia do Paraná, relacionadas ao Grupo Itararé, é atestada pela presença de numerosas superfícies de abrasão glacial. Inicialmente descritas no flanco oriental da Bacia do Paraná (Barbosa 1940, Almeida 1948, Bigarellaef et al. 1967, Rocha-Campos et al. 1968, 1988, Tomazelli \& Soliani 1982, 1997, Caetano-Chang et al. 1990 Campos 1992, Santos et al 1992) tais feicões foram recentemente reconhecidas no flanco ocidental da bacia (Gesicki 1996, Gesicki et al. 1996, 1998). De modo geral, as estrias e sulcos presentes nestas superfícies mostram uma direção média predominante segundo NNW, com indicações de movimento do gelo de sudeste para noroeste (Figura 1), tendo sido desenvolvidas sobre rochas do embasamento ou intraformacionalmente (v.g. Santos et al. 1996, Gesicki et al. 1996). Alguns autores admitem a existência de um centro glacial situado sobre o Arco de Assuncão, que teria sido o responsável pelos registros de sedimentação glacial presentes na parte ocidental da Bacia do Paraná (Crowell \& Frakes 1975, Santos et al. 1996). Entretanto, o paralelismo na direção das feições de abrasão glacial e a persistência no sentido de transporte do gelo têm sido argumentos favoráveis à hipótese de que as geleiras, provenientes de regiões situadas a sul-sudeste da Bacia do Paraná (sudoeste Africano), teriam atravessado o flanco oriental desta bacia e, durante os períodos de glaciação mais intensa, atingiram o seu flanco ocidental (Gesicki et al. 1996,1998).

No presente trabalho é descrita uma nova ocorrência de superfície estriada relacionada à glaciação neopaleozóica da Bacia do Paraná, localizada no Paraguai Oriental, e discutido seu significado paleogeográfico.

CONTEXTO GEOLÓGICO DA OCORRÊNCIA A superfície estriada está localizada na saída da localidade de Escobar (coordenadas $25^{\circ} 38^{\prime} 30^{\prime \prime} \mathrm{S}, 5^{\circ} 01^{\prime} 52^{\prime \prime} \mathrm{W}$ ), na estrada para Sapucai (Figura 2), em uma antiga área de empréstimo de cascalhes. No local ocorrem arenitos finos lamosos, avermelhados, contendo seixos geralmente dispersos, por vezes facetados e estriados. Karpoff (1965) reconheceu a origem glacial desses depósitos, aos quais referiu-se como Série Escobar, atribuindo-lhes idade pré-cambriana pelo fato de estarem situados em posição topográfica inferior em relação às unidades então consideradas como ordovicianas a silurianas. $\mathrm{O}$ autor estendeu esta designação a outras exposicõos de rochas situadas nos arredores de Paraguari, cuja origem glacial e idade carbonífera ou permo-carbonífera havia sido apontada em estudos anteriores (Harrington 1950, Eckel 1959).

Desde o reconhecimento do Rift de Assunção como uma das principais feições tectônicas do Paraguai Oriental (Degraff 1985) verificou-se que a região de Paraguari-Escobar-Sapucai está localizada no bloco baixo desta estrutura, próximo da sua borda norte. Tal fato permite constatar que os depósitos da Série Escobar são, na realidade, pós-silurianos, e podem ser apropriadamente enfeixados na Formação Aquidabán (e.g. Putzer 1962), de idade permo-carbonífera. Suas características litológicas e, em particular, a coloração avermelhada, são idênticas àquelas presentes nos depósitos da Formação Aquidabán e também parcialmente na Formação Coronel Oviedo, expostos na faixa de direção N-S que atravessa o Paraguai Oriental, partindo de Coronel Oviedo, ao sul, alcançando Bella Vista, ao norte (Fúlfaro 1996). A partir de Bella Vista esta faixa adentra o território brasileiro e os depósitos recebem a designação de Formação Aquidauana, lateralmente equivalente ao Grupo Itararé (e.g. Gesicki et al. 1998).

O bloco tectônico que preservou as exposições de rochas sedimentares permo-carboníferas em Escobar, além de estar tectonicamente rebaixado em relação aos blocos vizinhos, situados ao norte e ao sul do Rift de Assunção, sofreu também rotação horária, associada a tectonismo transcorrente dextral. Esta rotação ocorreu em dois episódios tectônicos distintos, o primeiro relacionado à instalação do rift, no Eocretáceo (Velázquez et al. 1998), e o segundo no Eoceno (Riccomini et al. 1998).

DESCRIÇÃO DA SUPERFÍCIE ESTRIADA O pavimento de Escobar é suborizontal, desenvolvido sobre o arenito e encontra-se inteiramente estriado. As estrias são paralelas, apresentam continuidade decimétrica e correspondem a sulcos de profundidade raramente superior a $1 \mathrm{~mm}$, com espaçamento de alguns milímetros, orientadas segundo N35E (Fig. 3). Em alguns locais a superficie está recoberta por diamictito compacto com seixos facetados e estriados (predomínio de quartito, quartzo e rochas granitóides), com espessura de até $20 \mathrm{~cm}$, distribuído de forma descontínua e com extensão horizontal não superior a $2 \mathrm{~m}$. Fraturas associadas à superficie encontram-se dispostas ortogonalmente às estrias e apresentam mergulhos para NNE (Fig. 4), correspondendo a fraturas transversais (transverse fractures segundo Boulton 1974) ou fraturas-em-crescente (crescentic fractures segundo Petit 1987, Eyles \& Boyce 1998). Outras feições associadas à superfície incluem seixos facetados (ferro-de-engomar) e estriados além de prováveis marcas-em-crescente.

DISCUSSÃO E CONCLUSÕES As características da superficie e sua associação com o diamictito - equivalente a um provável till de alojamento - indicam uma origem por abrasão de geleira. O padrão de distribuição das estrias, seu espaçamento e profundidade, aliado à presença de fraturas em crescente e de seixos facetados e estriados, são sugestivos de que o substrato, embora inconsolidado, apresentou comportamento coeso sob a ação dos processos de abrasão glacial; a elevada resistência ao cisalhamento decorre, provavelmente, do congelamento ou diagênese precoce do substrato, como verificado em algumas superfícies estriadas do Estado de Mato Grosso do Sul (Gesicki et al. 1996).

* Instituto de Geociências, Universidade de São Paulo, Caixa Postal 11.348,05422-970, São Paulo, SP, Fone: 55-11-818.4126, FAX: 55-11-818.4129, E-mail: riccomin@usp.br

** Bolsista de Produtividade em Pesquisa do Conselho Nacional de Desenvolvimento Científico e Tecnológico - CNPq

*** Bolsista de Pós-Doutorado da Fundação de Amparo à Pesquisa do Estado de São Paulo - FAPESP (Processo 97/00176-7)

Trabalho realizado com auxilio da FAPESP (Processos 94/03352-2 e 97/01210-4) 

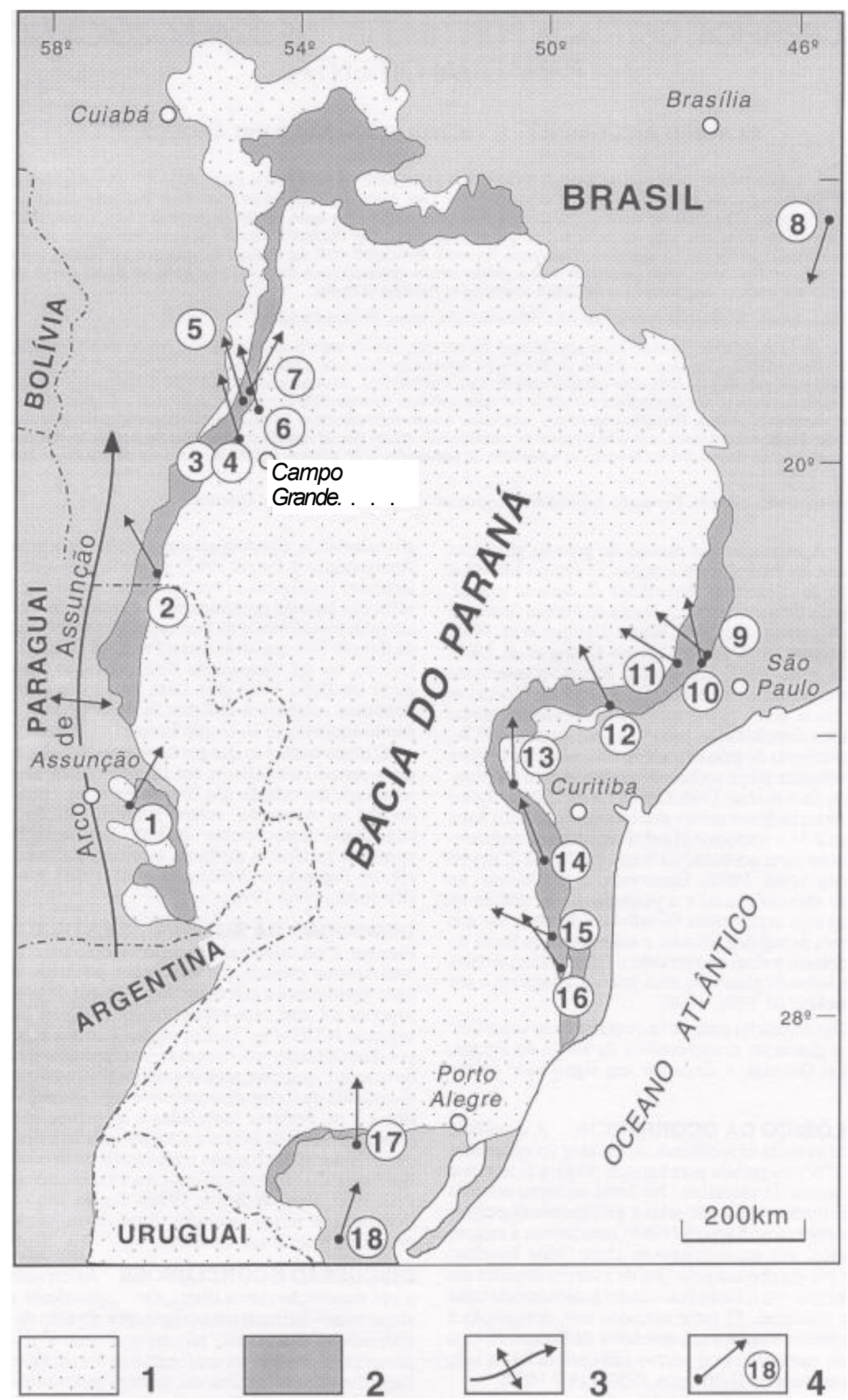

3

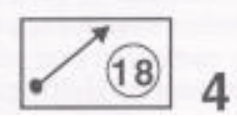

Figura 1 - Principais ocorrências de superficies estriadas e direções depaleofluxo de geleiras da glaciação neopaleozóica na Bacia do Paraná (modificado de Gesicki et al. 1996): 1 Bacia do Paraná; 2. Faixa de afloramentos do Grupo Itararé e Formação Aquidauana (Brasil) e formações Aquidabán e Coronel Oviedo (Paraguai); 3. Arco de Assunção; 4. Localização das principais superficies estriadas (l-Escobar, Paraguai; 2 - Rio Estrela, MS, Gesicki et al. 1996; 3 - Rio Aquidauana, MS, Gesicki et al. 1996; 4 - Ponte do Grego, MS, Gesicki et al. 1996; 5 - Rio Negro, MS, Gesicki et al. 1996; 6 - Serra Negra I, MS, Gesicki et al. 1996; 7 - Serra Negra 77, MS, Gesicki et al 1996; 8 - Santa Fé de Minas, MG, Campos 1992; 9 e 10 - Roche Moutonée de Salto, SP, Almeida 1948; 11 - pavimento de clastos de Jurumirim, SP, Rocha-Campos et al. 1968 ; 12 - Rio Piritubinha, SP, Caetano-Chang et al. 1990; 13 - Wittmarsum, PR, Bigarella et al 1967; 14 - Mafra, PR, Barbosa 1940; 15 - Alfredo Wagner, SC, Rocha-Campos et al. 1988; 16 - Trombudo Central, SC, Santos et al. 1992; 17 - Cachoeira do Sul, RS, Tomazelli \&Soliani Jr. 1982, 1997; 18 Pinheiro Machado, RS, Tomazelli \& Soliani Jr. 1982, 1997). 


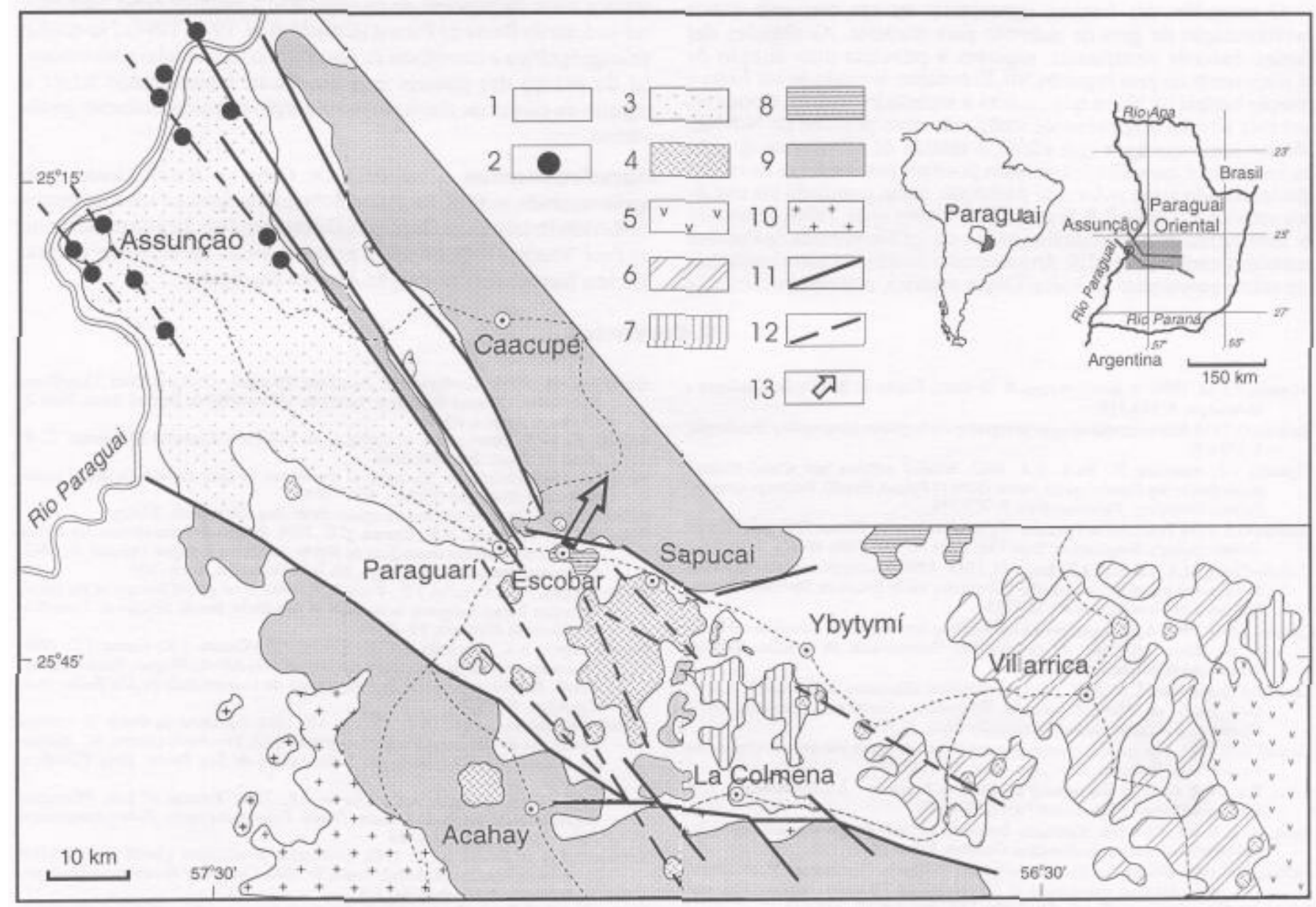

Figura 2 - Mapa geológico do Rift de Assunção (segundo Velázquez et al, 1998): L Coberturas sedimentares quaternárias; 2. Rochas alcalinas cenozóicas; 3. Depósitos cenozóicos da Formação Patino; 4. Rochas alcalinas cretáceas; 5. Derrames toleíticos eocretáceos da Formação Alto Paraná; 6. Arenitos eocretáceos da Formação Misiones; 7. Unidades sedimentares permianas do Grupo Independência; 8. Arenitos, diamictitos e folhelhos permo-carboniferos da Formação Aquidabán; 9. Unidades sedimentares ordovicianas-silurianas dos grupos Caacupé e Itacurubí; 10. Embasamento neoproterozóico; 11. Falhas principais; 12. Alinhamentos de rochas alcalinas; 13. Sentido de deslocamento do gelo na superficie estriada de Escobar.

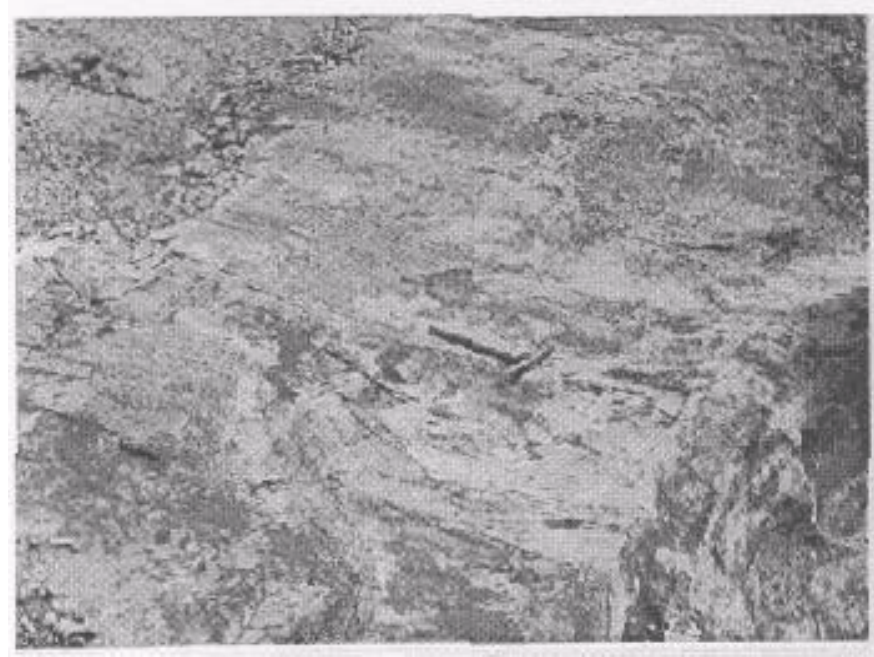

Figura 3- Aspecto geral da superficie estriada de Escobar, Paraguai Oriental, com estrias paralelas ao cabo do martelo.

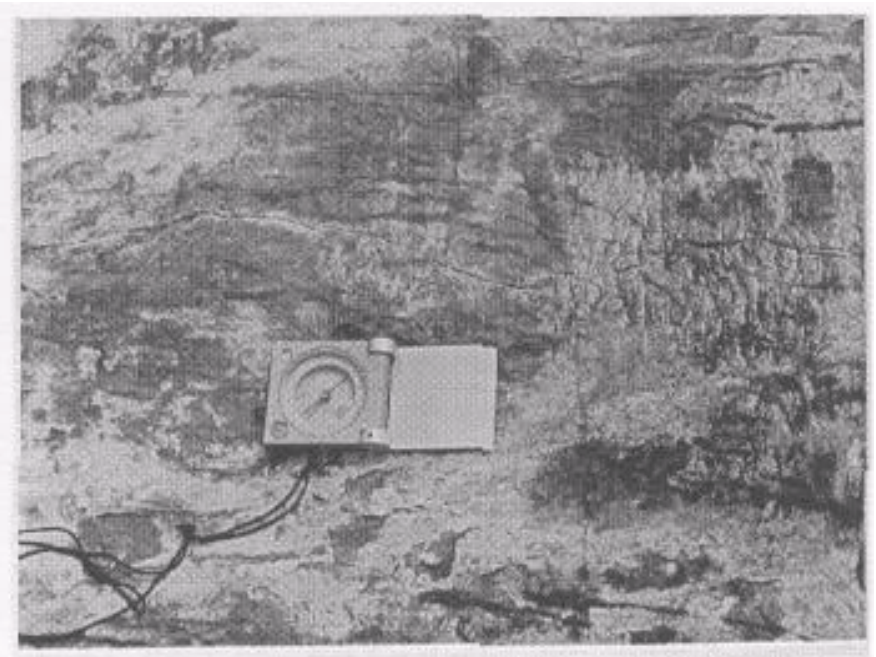

Figura 4 - Fraturas transversais ou em crescente, ortogonais às estrias (lado direito da foto). 
O mergulho das fraturas transversais ou em crescente indica movimentação do gelo de sudoeste para nordeste. As direções das estrias, bastante persistentes, sugerem a princípio uma direção de deslocamento do gelo segundo NE. Entretanto, levando-se em conta a rotação horária do bloco que contém a superfície estriada, é possível que esta direção originalmente tenha sido mais próxima de N-NNE. Mesmo sem considerar esse efeito, o sentido de movimento do gelo no local não é consistente com uma possível proveniência de centro glacial situado sobre o Arco de Assunção, como postulado em trabalhos anteriores (Crowell \& Frakes 1975, Santos et al. 1996). O sentido de movimento do gelo deduzido para a superfície estriada de Escobar mantém a tendência geral de deslocamento de sul para norte verificada em outras ocorrências da bacia. Dessa maneira, esta nova ocorrência soma a favor da hipótese de proveniência a partir de áreas situadas a sul-sudeste da Bacia do Paraná (Gesicki et al 1996,1998). No quadro paleogeográfico a ocorrência de Escobar é o testemunho mais ocidental do avanço das geleiras neopaleozóicas, representando talvez o registro do clímax da glaciação nesta porção do paleocontinente gondvânico.

Agradecimentos Ao Prof. Dr. Celso de Barros Gomes pelo apoio recebido, ao Prof. Dr. Paulo Roberto dos Santos e aos consultores da Revista Brasileira de Geociências pela revisão crítica do manuscrito, ao Prof. Thomas Rich Fairchild pelas sugestões apresentadas e a Sra. Thelma S amara pela confecção de parte das figuras.

\section{Referências}

Almeida, F.F.M. 1948. A Roche Moutonée de Salto, Estado de São Paulo. Geologia e Metalurgia, 5: 112-117.

Barbosa, 0.1940. Estrias produzidas por gelo permo-carbonífero. Mineração e Metalurgia, 4: $272-273$

Bigarella, J.J.; Salamuni, R.; Fuck, R.A. 1967. Striated surfaces and related features developed by the Gondwana ice sheets (State of Paraná, Brazil). Palaeogeography, Palaeodimatology, Palaeoecology, 3: 265-276.

Boulton, G.S. 1974. Processes and patterns of glacial erosion, In: Coates, D.R. (ed.) Glacial Geomorphology. Binghamton, State University of New York, 41-87.

Caetano-Chang, M.R.; Oliveira, J.P.; Brighetti, J.M.P. 1990. Pavimento estriado em rochas do Subgrupo Itararé ao longo do Rio Piritubinha, sul do Estado de São Paulo. Revista Brasileira de Geociências, 20: 333-335.

Campos, J.E.G. 1992. A glaciação permo-carbonifera nas regiões de Canabrava e Santa Fé de Minas, $M G$. Inst. de Geociências, Universidade de Brasília, Brasília, Dissertação de Mestrado, 104 p.

Crowell, J.C. \& Frakes, L.A. 1975. The Late Paleozoic Glaciation. In: Campbell, K.S.W. (ed.) Paper on Third Gondwana Symposium, Camberra, Austrália, 1973. Camberra, Australian National University Press, 313-331.

Degraff, J.M. 1985. Late mesozoic crustal extension and rifting on the western edge of the Paraná Basin, Paraguay. Geol. Soe. Atner. Abst., 17:560.

Eckel, E.B. 1959. Geology and mineral resources of Paraguay - A reconnaissance. U.S. Geological Survey Professional Paper, 327:1 10p.

Eyles, N. \& Boyce, J.I. 1998. Kinematic indicators in fault gouge: tectonic analog for soft-bedded ice sheets. Sedimentary Geology, 116:1-12.

Fúlfaro, V.J. 1996. Geology of Eastern Paraguay, In Comin-Chiaramonti, P. \& Gomes, C.B., eds., Alkaline magmatism in central-eastern Paraguay, relationships with coeval magmatism in Brazil. São Paulo, EDUSP/FAPESP, 103-122.

Gesicki, A.L.D. 1996. Geologia da Formação Aquidauana (Neopaleozóico, Bacia do Paraná) na porção centro-norte do Estado de Mato Grosso do Sul. Inst. de Geociências, Universidade de São Paulo, São Paulo, $126 \mathrm{p}$

Gesicki, A.L.D.; Riccomini, C.; Boggiani, P.C.; Coimbra, A.M. 1996. Evidências de avanço glacial na Formação Aquidauana (Neopaleozóico da Bacia do Paraná) no Estado de Mato Grosso do Sul. In: SBG, Congresso Brasileiro de Geologia, 39, Salvador, Anais, 2:124-127.

Gesicki, A.L.D.; Riccomini, C; Boggiani, P.C.; Coimbra, A.M. 1998. The Aquidauana Formation (Paraná Basin) in the context of Late Paleozoic glaciation in western Gondwana. Journal of African Earth Sciences, 27:81-82.
Harrington, H. 1950. Geologia del Paraguay Oriental. Contribuciones Científicas, Facultad de Ciências Exactas y Naturales, Universidad de Buenos Aires, Serie E: Geologia, Tomo I, 89p.

Karpoff, R. 1965. Observations géologiques au Sud-Est d'Asunción (Paraguay). C. R. Acad. Sc. Paris, 261:5558-5560.

Petit, J.-P. 1987. Criteria for the sense of movement on fault surfaces in brittle rocks. Journal of Structural Geology, 9:597-608

Putzer, H. 1962. Die geologie von Paraguay. Beitr. Re g. Geol. Erde, 2:182p.

Riccomini, C.; Velázquez, V.F.; Gomes. C.B. 1998. Padrão de fraturamento das rochas alcalinas ultramáficas cenozóicas do Rift de Assunção, Paraguai Oriental. In: SBG Congresso Brasileiro de Geologia, 40, Belo Horizonte, Anais, 109.

Rocha-Campos, A.C.; Farjallat, J.E.; Yoshida, R. 1968. New glacial features of the Upper Paleozoic Itararé Subgroup in the state of São Paulo, Brazil. Boletim da Sociedade Brasileira de Geologia 17: 47-57. Rocha-Campos, A.C.; Machado, L.C.R.

Santos, P.R.; Canuto, J.R.; Castro, J.C. 1988.

Pavimento estriado da glaciação neopaleozóica em Alfredo Wagner, Santa Catarina, Brasil. Boletim do Instituto de Geociências da Universidade de São Paulo, Série Cientifica, 19: 39-46.

Santos, P.R.; Rocha-Campos, A.C.; Canuto, J.R. 1992. Estruturas de arrasto de icebergs em ritmito do Subgrupo Itararé (Neopaleozóico), Trombudo Central, SC. Boletim do Instituto de Geociências da Universidade de São Paulo, Série Científica 23:1-18. Santos, P.R.; Rocha-Campos, A.C.; Canuto, J.R. 1996. Patterns of Late Palaeozoic deglaciation in the Paraná Basin, Brazil. Palaeogeography, Palaeodimatology, Palaeoecology, 125: 165-184.

Tomazelli, L.J. \& Soliani Jr., E. 1982. Evidências de atividade glacial no Paleozóico Superior do Rio Grande do Sul, Brasil. In: SBG, Congresso Brasileiro de Geologia, 32, Salvador, Anais, 4:1378-1391. Tomazelli, L.J. \& Soliani Jr., E. 1997.

Sedimentary fácies and depositional environments related to Gondwana Glaciation in Batovi and Suspiro regions, Rio Grande do Sul, Brazil. Journal of South American Earth Sciences, 10:295-303.

Velázquez, V.F.; Riccomini, C.; Gomes, C.B. 1998. Tectônica Mesozóica no Rift de Assunção, Paraguai Oriental. In: SBG, Congresso Brasileiro de Geologia, 40, Belo Horizonte, Anais, 112.

Manuscrito A-1029 Recebido em IS de setembro de 1998 Revisão dos autores em 20 de janeiro de 1999 Revisão aceita em 25 de janeiro de 1999 